\title{
Experimental Study on the Connection of Nigral Apoptosis of Normal Rats and Rats of Parkinson Disease with Different Doses of Levodopa
}

Cao Fei $^{1 *}$, Sun Sheng-Gang ${ }^{1}$, Cao Xue-Bing ${ }^{1}$, Wang Tao ${ }^{1}$, Cheng Ji-Xiang ${ }^{1}$, Mei Yuan-Wu' ${ }^{1}$, Tong E-Tang ${ }^{1}$ and Luo Fang ${ }^{2}$

${ }^{1}$ Department of Neurology, Union Hospital, Tong-ji Medical College, Hua-zhong Scientific and Technologic University, Wuhan, PR China

${ }^{2}$ Trade and Financial Hospital of Hubei Province, Wuhan, PR China

\begin{abstract}
Objective: To evaluate the neuronal toxicical effects induced by levodopa and explore the method in which PD can be treated reasonably with levodopa.

Method: The rat models of Parkinson disease (PD) were made by injecting stereotaxically 6-OHDA to right side of the mesencephic ventral tegmental area (VTA) and substantia nigra pars compacta (SNc). The PD rats were treated intraperitoneally with different dosages $(10 \mathrm{mg} / \mathrm{kg} / \mathrm{d}, 50 \mathrm{mg} / \mathrm{kg} / \mathrm{d}, 100 \mathrm{mg} / \mathrm{kg} / \mathrm{d})$ and different durations $(1 \mathrm{~d}, 3 \mathrm{~d}, 5 \mathrm{~d}, 7 \mathrm{~d})$ of levodopa. At the same time, we observed the injury of substantia nigral by the way of treating PD rats with levodopa for 7 days and then stopped for 7 days. In this study, we used TUNEL to detect the injury of substantia nigra by different doses and durations of levodopa.
\end{abstract}

Results: The number of substantia nigral apoptosis was increased with the changes of doses and durations of levodopa.

Conclusions: If we use levodopa intermittently or as little as possible, the toxical action of levodopa may be reduced.

Keywords: L-dihydroxy-phenylalanine (L-dopa); Apoptosis

\section{Introduction}

Parkinson disease is a common in middle-aged and elderly chronic degenerative diseases of central nervous system. Current clinical practice is still represented by L-dopa dopamine replacement therapy mainly, some researchers $[1,2]$. Consider L-dopa has a role in neurotoxicity, recent studies [3] suggest that PD may be through apoptosis which caused a common path. L-dopa can increase the PD substantia nigra in rats the role of apoptosis still controversial, whether the role of dose-related has not been reported. The study subjects through the act of observation, the number of substantia nigra cell apoptosis detection, in situ hybridization study of different doses of L-dopa in rat substantia nigra of PD cell toxicity and to explore the role of the toxicity mechanism of gene regulation, so as to clinical more reasonable use of L-dopa treatment of Parkinson's disease and provide experimental basis.

\section{Materials and Methods}

\section{Preparation of rat model of PD}

Healthy male Wistar rats were purchased from Animal Center of Tongji Medical University, The animals weighed between 180 to 200 $\mathrm{g}$ and were 3-months-old. Using improved Thomas methods [4] the production of $\mathrm{PD}$ rat model and validate the success model.

\section{Animal selection and grouping}

Select the success of PD rat model 100, were randomly divided into two major groups: PD group $(n=25)$, L-dopa group $(n=75)$. L-dopa group of PD rats 8:00 daily intraperitoneal injection of L-dopa injection: (L-dopa injection: L-dopa do powder (Sigma product) dissolved in ethanol containing $0.05 \%$ and $0.1 \%$ ascorbic acid (Sigma product) water for injection, the preparation of $10 \mathrm{mg} / \mathrm{ml}, 50 \mathrm{mg} /$ $\mathrm{ml}, 100 \mathrm{mg} / \mathrm{ml}$ in three concentrations of L-dopa injection) To the observation point, according to their points of small doses $(10 \mathrm{mg} /$ $\mathrm{kg} / \mathrm{d})$, medium $(50 \mathrm{mg} / \mathrm{kg} / \mathrm{d})$, large $(100 \mathrm{mg} / \mathrm{kg} / \mathrm{d})$ three group $(\mathrm{n}=25)$; PD group was injected in addition to L-dopa from L-dopa injection of other components. Observation point for the $1 \mathrm{~d}, 3 \mathrm{~d}, 5 \mathrm{~d}, 7 \mathrm{~d}, 14 \mathrm{~d}, 14$ $\mathrm{d}$ for the PD dose in each group of rats by intraperitoneal injection of L-dopa injection $\times 7 \mathrm{~d}$, drug $7 \mathrm{~d}$.

Each group per time point 5 PD rats. The control group according to the above point to give L-dopa $100 \mathrm{mg} / \mathrm{kg} / \mathrm{d}$.

\section{Outcome Measures}

\section{Detected behavior}

The control group, L-dopa group were defacto the second day of the scheduled 8:00 behavioral observation study, Intraperitoneal injection of APO $0.5 \mathrm{mg} / \mathrm{kg}, 40 \mathrm{~cm}$ in diameter stainless steel basin Observation of rotational behavior in rats, Measurement of rotation number. Then perfusion fixed, get in the brain, paraffin-embedded.

\section{Apoptosis detect (TUNEL method)}

Seaton by ways apoptotic cells in the substantia nigra line detection [5].

\section{Image Analysis}

The use of multimedia BUAA Photo True Color pathological analysis system for image analysis of biopsy. All the slices are of uniform magnification $(100 \times)$, under the same light intensity analysis, each case of apoptosis, in situ hybridization for the immediate vicinity of the three sections, the substantia nigra area to observe the same size, measure the window for $262144 \mu \mathrm{m}^{2}$, analysis of indicators:

*Corresponding author: Cao Fei, Department of Neurology, Union Hospital, Tongji Medical College, Huazhong University of Science and Technology, Wuhan, PR China, E-mail: caofyx@163.com

Received April 11, 2013; Accepted July 28, 2013; Published July 31, 2013

Citation: Fei C, Sheng-Gang S, Xue-Bing C, Tao W, Ji-Xiang C, et al. (2013) Experimental Study on the Connection of Nigral Apoptosis of Normal Rats and Rats of Parkinson Disease with Different Doses of Levodopa. J Neurol Disord 2: 125. doi:10.4172/2329-6895.1000125

Copyright: (C) 2013 Fei C, et al. This is an open-access article distributed under the terms of the Creative Commons Attribution License, which permits unrestricted use, distribution, and reproduction in any medium, provided the original author and source are credited. 


\begin{tabular}{|c|c|c|c|c|c|}
\hline Control group & 45 & 54 & 51 & 50 & 48 \\
\hline PD group & $407 \pm 32^{\star}$ & $400 \pm 29^{\star}$ & $388 \pm 26^{\star}$ & $368 \pm 20^{\star}$ & $174 \pm 15^{\star}$ \\
\hline Low-dose group & $412 \pm 35^{\star}$ & $418 \pm 26^{\star}$ & $409 \pm 23^{\star}$ & $403 \pm 24^{\star}$ & $226 \pm 22 \star *$ \\
\hline Medium dose group & $468 \pm 33^{\star} \approx \diamond$ & $533 \pm 49 \star \approx \diamond$ & $690 \pm 45^{\star \Delta \diamond}$ & $605 \pm 37^{\star} \Delta \diamond$ & $320 \pm 22^{\star *}$ \\
\hline High-dose group & $759 \pm 61^{\star} \triangle \Delta \diamond$ & $647 \pm 47^{\star} \triangle \Delta \diamond$ & $577 \pm 49 \star \Delta \triangleleft \diamond$ & $486 \pm 37^{\star} \triangle \Delta \diamond$ & $386 \pm 20 \star \Delta \Delta$ \\
\hline
\end{tabular}

$\star$ Compared with the control group $p<0.05$

※ith the PD group $p<0.05$

$\triangle$ With the PD group $p<0.01$

$\diamond$ With low-dose group $\mathrm{p}<0.05$

$\Delta$ Compared with the middle dose group $p<0.05$

Table 1: The normal and PD substantia nigra in rat apoptotic changes (unit: quantity $/ \mathrm{mm}^{2}$ ).

positive number density, the positive surface density. All data analysis system are automatically calculated and printed.

\section{Statistical Methods}

All data are expressed as mean \pm standard deviation $(\mathrm{X} \pm \mathrm{SD})$ said that the use of mathematical statistics Oxstat group process is complete, the group paired data test of significance (ANOVA).

\section{Results}

\section{L-dopa-induced cell apoptosis in the outcome of PD in rats}

PD group the number of nigral apoptosis gradually decreased with time; small dose group $1 \mathrm{~d}, 3 \mathrm{~d}$ little change, $5 \mathrm{~d}, 7 \mathrm{~d}$ decline, 14 $\mathrm{d}$ group the lowest, with the exception of $14 \mathrm{~d}$, the other the defacto with the PD group was no significant difference; Medium dose Group apoptosis $14 \mathrm{~d}<1 \mathrm{~d}<3 \mathrm{~d}<7 \mathrm{~d}<5 \mathrm{~d}, 5 \mathrm{~d}$ highest point of time than the $P D$ group, low-dose group was significantly higher; the number of highdose group apoptosis $14 \mathrm{~d}<7 \mathrm{~d}<5 \mathrm{~d}<3 \mathrm{~d}<1 \mathrm{~d}, 1 \mathrm{~d}$ highest the defacto high-dose group than the small number of apoptotic, middle dose group and PD group was significantly increased (Table 1).

\section{Discussion}

(a) In L-dopa dopamine replacement therapy are the main drug, its side effects are also very obvious that the latest studies suggest that L-dopa has resulted in the substantia nigra apoptosis [6,7]. High-dose group the peak of apoptosis in the $1 \mathrm{~d}, 3 \mathrm{~d} \sim 7 \mathrm{~d}$ decreased 7 days after discontinuation of treatment, decreased significantly close to the control of water found in this study low-dose L-dopa group apoptotic with the control group no significant difference; Medium dose group number of apoptotic nigral with L-dopa treatment time increases, the peak appears in the 5 days, and 7 days after discontinuation of apoptotic down to the level of the control group level; at the same time point in each group the number of substantia nigra cell apoptosis is also with the L-dopa dose increased.

The above results indicate that cell apoptosis from the perspective of L-dopa with proven neurotoxic effects, apoptosis involved in the L-dopa on the injury and the role of stromal cells. L-dopa can accelerate apoptosis in the substantia nigra. L-dopa nerve toxicity with the drug dose, drug use increased with increasing time. L-dopa treatment in each group 7 days after discontinuation of treatment, apoptosis was significantly reduced and shows that L-dopa neurotoxicity are reversible, drug can reduce the toxic effects.

(b) L-dopa neurotoxicity is not self-caused, but its decomposition products arising from oxidative metabolism [8]: (1) L-dopa oxidation can generate its own superoxide dismutase, hydrogen peroxide, hydroxyl radical, quinones, semiquinone radicals, etc., can be lead to PC12 cell apoptosis or death, even if L-dopa to DA conversion is inhibited, the toxic effects still exist and can be inhibited antioxidants
[7]. (2) Inhibition of substantia nigra mitochondrial enzyme complex I (Complex I) [9]. (3) DNA can cause substantial damage to nerve cells [10]. This experiment found that L-dopa in rat substantia nigra of normal non-induced cell apoptosis. Jenner thinks [11] long-term oral L-dopa does not nigrostriatum of normal damage, but it can add to have damage in the substantia nigra striatal damage, but there is no sufficient basis for the clinical experimental evidence confirmed that L-dopa to dopamine neurons have residual damage, probably because the substantia nigra of normal rat striatum system perfect free radical scavenging system, a reasonable distribution of iron ions are reasonable, the generation of free radicals and metabolism are reasonable, and metabolism of toxic decomposition product has a certain ability to carry out rapid metabolism.

About PD treatment, researchers have advocated the early use of dopamine receptor agonist, but clinical data show that only $30 \%$ of patients effectively, and the existence of consciousness vague, visual hallucination, hemianopsia such as side effects, so the traditional DA replacement therapy is still the main means of treatment of PD The key is how to reach best effect and least side-effects. Through this experiment, we believe that: (1) Early PD, in order to reduce the decomposition products from DA-induced neurotoxicity may be taken monoamine oxidase inhibitors or drugs such as anti-free radical: Amphetamine-Propargyl, vitamin E, vitamin A such that DATATOP program (deprenyl and tocophenol antioxidation therapy of parkinsonism). (2) L-dopa treatment are advised to start small doses, steady and high-dose medication advocates multiple use and minimize the dose of each medication, medication to extend the time interval. (3) L-dopa controlled release tablets containing simultaneously inhibit L-dopa, DA decomposition of pharmaceutical ingredients is an ideal method of treatment, researchers have found that continuous DA stimulation of long-term treatment has less complications than fluctuation in long-term treatment.

\section{References}

1. Henry B, Crossman AR, Brotchie JM (1998) Characterization of enhanced behavioral responses to L-dopa following repeated administration in the 6 -hydroxydopamine lesioned rat model of Parkinson disease. Exp Neurol 151: 334-342.

2. Mena M A, Pardo B, Casarejos M J, Fahn S, García de Yébenes J (1992) Neurotoxicity of levodopa on catecholaminergic neurons. Mov Disord 7: 23-31.

3. Walkinshaw G, Waters CM (1994) Neurotoxin-induced cell death in neuronal PC12 cells is mediated by apoptosis. Neuronscience 63: 975-987.

4. Thomas J, Wang J, Takuba H, Sheng J, de Jesus S, et al. (1994) A 6 -OHDA induced selective Parkinsonian rat model: further biochemical and behavioural characterization. Exp Neurol 126: 159-167.

5. Seaton AT, Cooper JM, Schapira AH (1998) Cyclosporin inhibition of apoptosis induced by mitochondrial complex I toxins. Brain Res 809: 12-17.

6. Masserano JM, Gong L, Kulaga H, Baker I, Wyatt RJ (1996) Dopamine induced apoptosis cell death of a catecholaminergic cell line derived from the central nervous system. Mol Parmacol 50: 1309-1315. 
Citation: Fei C, Sheng-Gang S, Xue-Bing C, Tao W, Ji-Xiang C, et al. (2013) Experimental Study on the Connection of Nigral Apoptosis of Normal Rats and Rats of Parkinson Disease with Different Doses of Levodopa. J Neurol Disord 2: 125. doi:10.4172/2329-6895.1000125

Page 3 of 3

7. Melamed E, Offen D, Shirvan A, Djaldetti R, Barzilai A, et al. (1998) Levodopa toxicity and apoptosis. Ann Neurol 44: 149-154.

8. Zou L, Jankovic J, Rowe DB, Xie W, Appel SH, et al. (1999) Neuroprotection by pramipexole against dopamine- and levodopa- induced cytotoxity. Life Sci 64: $1275-1285$.

9. Offen D, Ziv I, Sternin H, Melamed E, Hochman A (1996) Prevention of dopamine induced cell death by thiol antioxidants: possible implications for treatment of Parkinson disease. Exp Neurol 141: 32-39.

10. Spencer JP, Jenner A, Aruoma OI, Evans PJ, Kaur H, et al. (1994) Intense oxidative DNA damage promoted by L-dopa and its metabolites implications for neurodegenerative disease. FEBS Lett 353: 246-250.

11. Jenner PG, Brin MF (1998) Levodopa neurotoxicity: experimental studies versus clinical relevance. Neurology 50: 39-43. 